\title{
The vehicle routing problem in urban networks: an approach based on a network fundamental diagram
}

\author{
G. Musolino ${ }^{1}$, A. Polimeni ${ }^{2}$ \& A. Vitetta ${ }^{1}$ \\ ${ }^{1}$ DIIES - Dipartimento di ingegneria dell'Informazione, \\ delle Infrastrutture e dell'Energia Sostenibile, \\ Università degli Studi Mediterranea di Reggio Calabria, Italy \\ ${ }^{2}$ DICIEAMA - Dipartimento di Ingegneria Civile, Informatica, \\ Edile, Ambientale e Matematica Applicata, \\ Università degli Studi di Messina, Italy
}

\begin{abstract}
This paper presents a formulation of the vehicle routing problem (VRP) based on the concept of a network fundamental diagram (NFD). The proposed model for estimating the (weighted) link costs considers variables describing the average traffic conditions related to homogeneous portions of the network. They are synthetized in a NFD. The proposed VRP computes the optimum path between each couple of clients by taking into account link costs and average values of density, which may be considered as proxies of travel time reliability. An application to a real dimension network allowed the testing of the proposed method.

Keywords: vehicle routing problem, network fundamental diagram, timedependent link costs, genetic algorithm.
\end{abstract}

\section{Introduction}

The vehicle routing problem (VRP) has been object of several studies in literature and many solution procedures were proposed in last years (see [1]). Dantzig and Ramser [2] presented in the seminal paper the first VRP formulation: the problem was called a truck dispatching problem and the aim was to mimimize the total distance traveled by trucks. Later, the problem was extended and reformulated to 
take into account different elements related with the freight delivery/pick-up operations. As an example (the papers in this field are several, in this work only few oh them are recalled), some problem formulations consider constraints in the freight delivery (pick-up) time period $[3,4]$. In other formulations $[5,6]$, travel demand can change during the travel time period (may be necessary to change route), or the delivery and pick-up operations are made jointly [7, 8].

In many cases the proposed approaches are based on static estimation of costs (in term of travel time), and only in few cases the estimation of costs is based on traffic flow theory and/or on traffic system simulation in real time [9-11]. Advancements in VRP formulation consider time-dependent evolution of the network [12-14], where link costs (to use as input in VRP) are obtained by means of a (static or dynamic) assignment procedure.

The paper proposes a formulation of the VRP based on the concept of network fundamental diagram (NFD). In traffic flow theory, the NFD [15, 16] correlates global values of vehicular flows and densities. In some cases, the assignment models $[17,18]$ may support the estimation of link costs, considering also their structural conditions [19].

An enhancement proposed in this paper concerns the definition of NFDs associated to a homogenous portions of the network, obtained after a zoning of the study area. The aim is to estimate an average value of density related to each portion, which may be considered as a proxy of travel time reliability. Moreover, link costs are evaluated for each time slice (e.g. 10 minutes) of the total period of analysis (e.g. hours). The VRP is formulated in order to minimize the total travel time, as the sum of (weighted) link travel times estimated by taking into account the above elements. An application on a real dimension problem is proposed in order to evaluate the method.

The paper is articulated as follows. Section 2 reports the VRP formulation. Section 3 presents an application to an urban network of real dimensions. The last section concerns the research perspectives.

\section{Problem formulation}

This section presents the proposed problem formulation reporting the assignment procedure, the link costs formulation and the routing problem.

The main inputs of our formulation are (Figure 1): the transport network, a study area divided into homogenous zones, a NFD for each portion of the network. The NDFs are estimated from data obtained from a microscopic traffic simulator (the expected value of link cost is provided). Link costs are estimated; considering, for each zone, the NFD and the time slice. Particularly, link costs are a function of the expected costs and of a variance depending on the cost distribution in the time. Then, a path search algorithm (Dijkstra algorithm) finds the best paths on the network, considering that the path cost depends on time slice and zone. Finally, a vehicle routing optimization model identifies the best routes for freight vehicles considering the time variability of path costs with a discrete approach. In [20] the problem is solved considering continuous cost functions. 


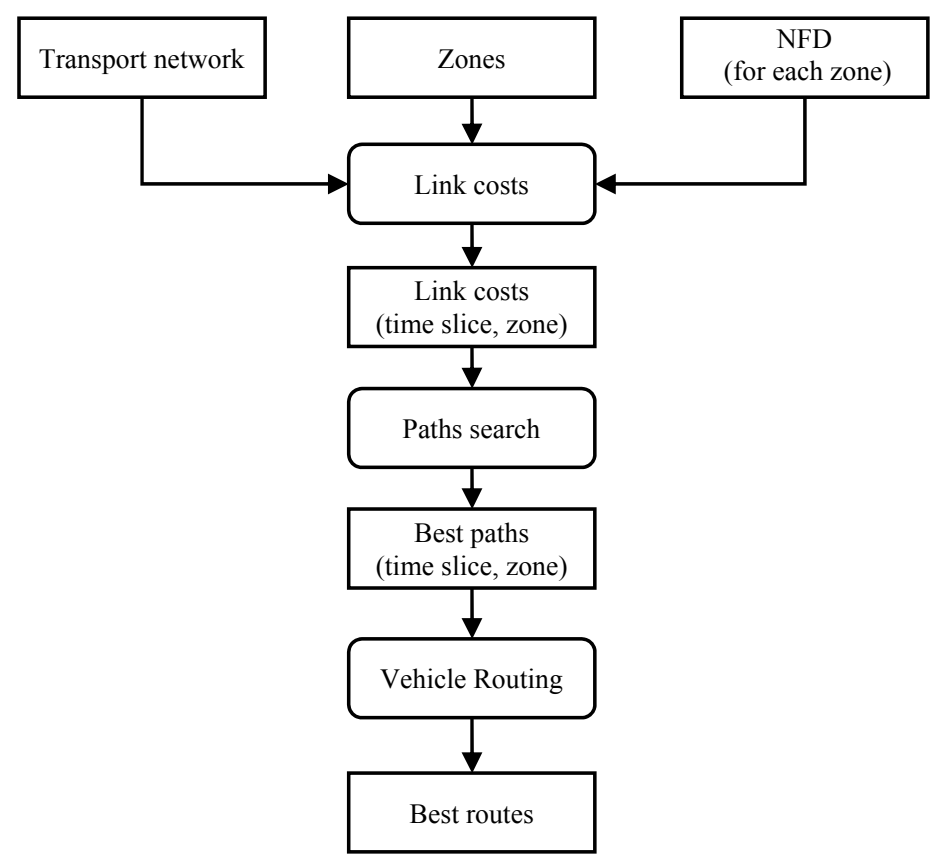

Figure 1: Flowchart of the proposed formulation.

\subsection{Network Fundamental Diagram (NFD)}

Two classes of vehicle are considered: cars and freight vehicles. Four indicators are specified in order to build the NFD [15, 16, 21]:

- $\quad$ Total travel distance (TTD)

$$
T T D=\sum_{i} f_{j} \cdot l_{j} \quad j \in A
$$

- $\quad$ Total number of vehicles (TNV)

$$
T N V=\Sigma_{j} k_{i} \cdot l_{j} \quad j \in A
$$

- $\quad$ Average flow (AF)

$$
A F=\Sigma_{j} f_{j} \cdot l_{j} / L \quad j \in A
$$

- $\quad$ Average density (AD)

$$
A D=\Sigma_{j} k_{j} \cdot l_{j} / L \quad j \in A
$$


with (for each link $j$ ):

$f_{j}$, vehicular flow;

$k_{j}$, vehicular density;

$l_{j}$, link length;

$L=\Sigma_{\mathrm{j}} l_{j}$, total network length;

$A$, link set.

The above indicators can be evaluated for the entire network or for a portion of it; in the second case links $\mathrm{j}$ considered in the indicators evaluation belong to a specific zone $\left(j \in A^{\prime}\right.$ with $\left.A^{\prime} \subseteq A\right)$.

It is possible to define a critical value of $A D\left(A D^{c r}{ }_{z}\right.$ in Figure 2$)$ as the value of the density corresponding to the maximum value of flow $A F^{\max }{ }_{z}$. As the NFD may be related to a zone of the urban area, it is possible to define a set $B=\left\{\ldots A D^{c r}{ }_{z}, \ldots\right\}$ containing the values of critical density for each zone $z \in Z$ (being $Z$ the set of the zones).

Moreover, for each time slice, each zone is characterized by a couple of flowdensity values in the NFD (an example is reported in the Figure 2).

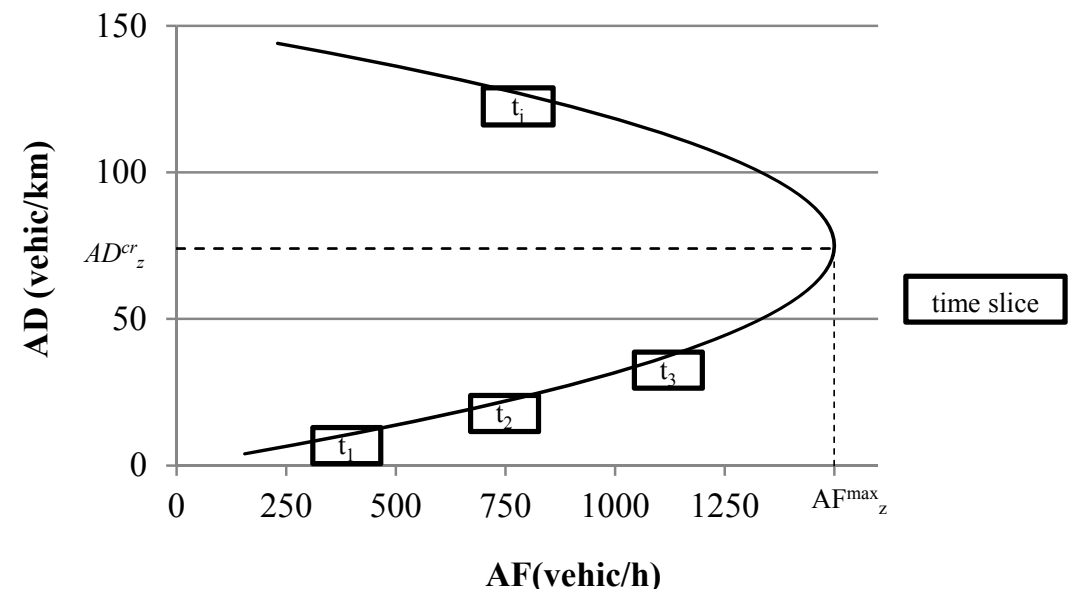

Figure 2: Schematic plot of the NFD.

\subsection{Link costs}

The dynamic traffic assignment (DTA) model provides, for each link $j$ and for each time slice $m$, a value of the expected link cost $\overline{c_{m j}}\left(\overline{c_{m j}}\right.$ is the expected value of the link cost distribution).

The weighted link cost is evaluated as a function $(\varphi)$ of the expected value of the $\operatorname{cost} \overline{c_{m j}}$ and the relative variance $\sigma_{m j}$, assumed as a function of the link density $k_{m j}$ :

$$
\widehat{c_{m j}}=\varphi\left(\overline{c_{m j}}, \sigma_{m j}, k_{m j}\right)
$$


A specification of eq. (1) may be reported in the following:

$$
\widehat{c_{m j}}=\overline{c_{m j}} \cdot\left(1+\beta_{1} \cdot k_{m j} / k^{c r}+\beta_{2} \cdot A D_{m z} / A D^{c r}\right)
$$

where

$k_{m j}$ is the density of link $j$ in the time slice $m$;

$k^{c r}{ }_{j}$ is the critical density according to the macroscopic flow-density diagram of link $j$;

$A D_{m z}$ is the average density for network portion of zone $z$ in time slice $m$ according to the NFD;

$A D^{c r}{ }_{z}$ is the critical density for network portion of zone $z$ according to the NFD;

$\beta_{1}$ and $\beta_{2}$ are parameters.

In time slice $m$, the term $\beta_{1} \cdot k_{m j} / k^{c r}{ }_{j}$ of Eq. (2) takes into account the reliability of link cost due to the local traffic conditions on the link; the term $\beta_{2} \cdot A D_{m z} / A D^{c r}{ }_{z}$ takes into account the reliability of the link cost due to the average traffic conditions related to the portion of the network belonging to the zone.

According to this approach, it is necessary to estimate a value of link cost for each time slice. This means that when a vehicle travel on the network if changes the time slice it is necessary to re-computing the shortest paths.

\subsection{Routing}

The vehicle routing problem is formulated with the aim of minimizing the total travel cost for freight vehicles. The assumptions are:

1) weighted link costs are estimated by means of Eq. (2),

2) path cost is weighted during the travel, considering the variation of the link costs varying the time slice.

In this way, the cost $g_{k(r, s)}$ of a path $k_{(r, s)}$ between origin $r$ and destination $s$ is:

$$
g_{k(r, s)}=\sum_{j} \delta_{k j m} \cdot \zeta\left(\widehat{c_{m j}}\right)
$$

where:

$\delta_{k j m}$ is equal to 1 if the link $j$ belong to the path $k$, in the time slice $m ; 0$ otherwise; $\zeta($.) is a function to identify the time slice in which estimate the link cost.

The formulated objective function is an extension of the one presented in [14]:

$$
\Phi(X)=\Sigma_{v}\left(\sum_{r} \Sigma_{s}\left(g_{k(r, s)}\right) \cdot x_{k(r s) v}\right)
$$

where:

$v$ is a generic vehicle;

$(r, s)$ is the origin destination pair;

$x_{k(r s) v}$ is the design binary variable (is equal to 1 if the vehicle $v$ use the path $k_{(r, s)} 0$ otherwise). 
The constraints of the problem, as reported in [11], are:

- $\quad$ only one vehicle can visit a node;

- all vehicles leave from the depot and come back to it;

- capacity constraint;

- the variable $x_{k(r s) v}$ can assume the value zero or one.

\section{Application}

This section reports an application of the routing problem (4) to the network of Villa San Giovanni, a town in the South Italy. The network has 359 links and 136 nodes (Figure 3). The study area is subdivided into three zones (A, B and C): a NFD (Figure 4) and a critical value of critical density $A D^{c r}{ }_{z}$ are available [21] for each zone $z$.

Figure 4 shows the scatterplots between the estimated values of $\mathrm{AF}$ and $\mathrm{AD}$ for each of the three zones of the study area. Moreover, the values of $A D^{c r}{ }_{z}$ are reported. It emerges that zone $\mathrm{B}$ presents values of $\mathrm{AF}$ and $A D^{c r}{ }_{z}$ greater than the ones of zones $\mathrm{A}$ and $\mathrm{C}$. This is due to the fact that zone $\mathrm{B}$ covers the central part of the town with higher levels of congestion.

The origin-destination demand matrix is obtained by means of a survey [22]; average trips per hour in the study area are 3631 (vehicles/hour), with 1627 trips with internal origins and destinations and 469 trips having external origins and/or destinations.

The clients to be served are 28 (named from 2 to 29 in Figure 4) and they are located in the three zones of the study area. The depot is in the node 1 . The optimization procedure is based on a genetic algorithm, assuming a fleet of two homogeneous freight vehicles that travel from the depot to the clients and three time slices. A Dijkstra algorithm is used to find the optimum path between each couple of clients [23].

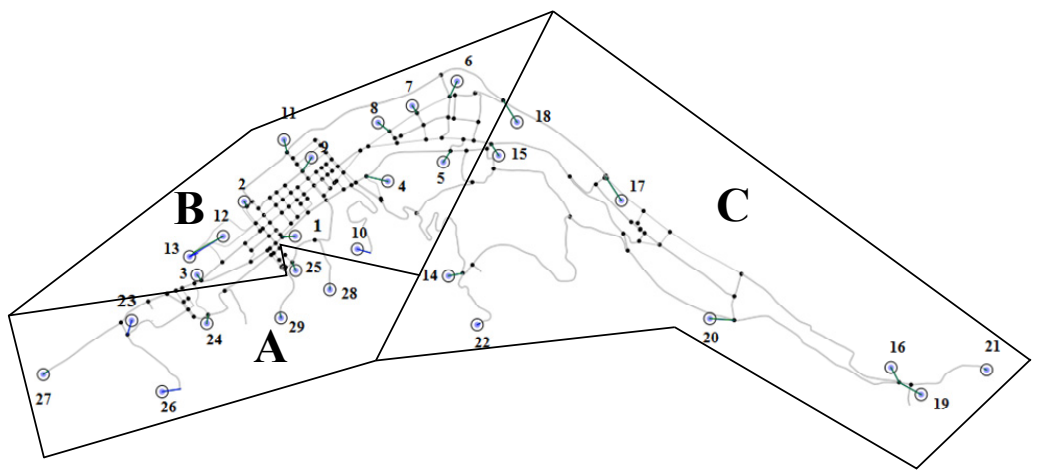

Figure 3: Urban network of Villa San Giovanni and identification of three zones. 


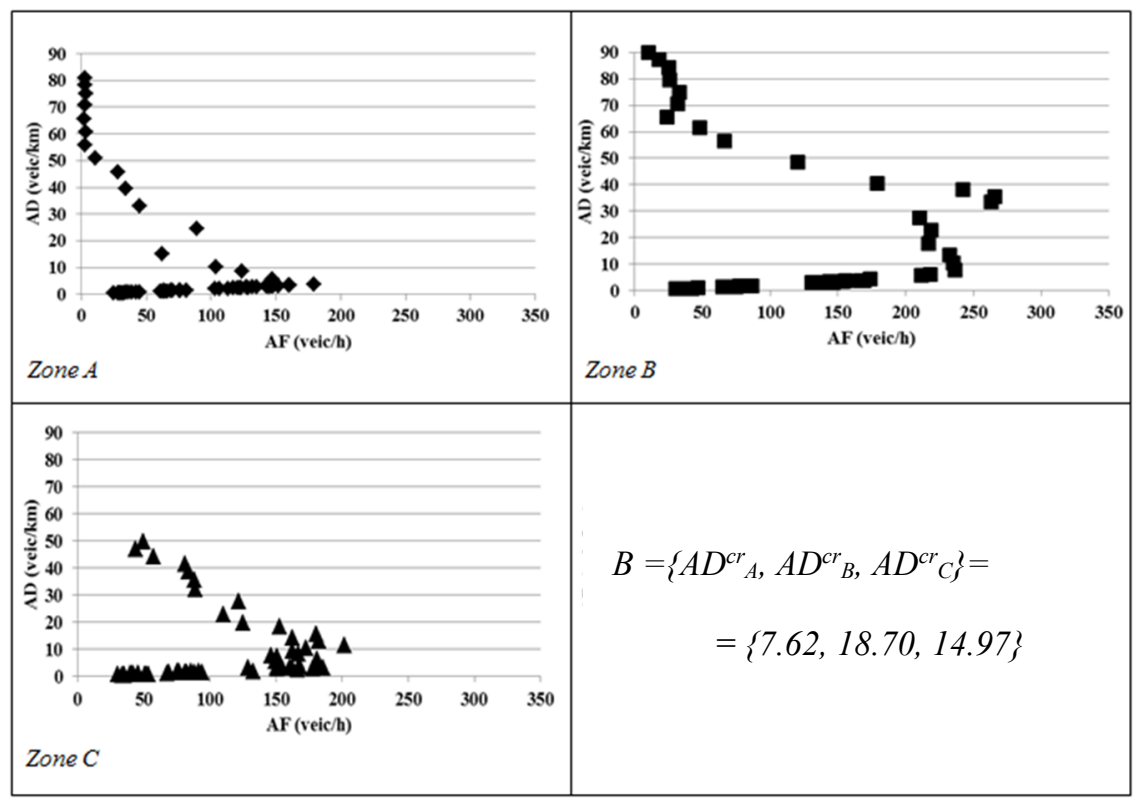

Figure 4: $\quad$ NFD for each zone and values of set $B$ [21].

The values of $A D_{m z}$ are estimated for each zone $z$ and time slice $m$. The value of $A D_{m z}$ is major or minor than the critical value $A D^{c r}{ }_{z}$, according to the NFD defined for each zone. The preliminary results obtained are reported in Table 1.

The optimal solution consists of two routes with sequence of clients, expected and weighted costs presented in Table 2 .

The optimization procedure tends to find a solution in which clients belonging to a zone $z$ are visited in a time slice where $A D_{z}<A D^{c r}{ }_{z}$.

Table 1: Average density vs. critical density for each time slice and zone.

\begin{tabular}{cccc}
\hline Zone $z$ & Time slice $m$ & 1 & 2 \\
\hline $\mathrm{A}$ & $A D_{I A}<A D^{c r}{ }_{A}$ & $A D_{l A} \geq A D^{c r}{ }_{A}$ & $A D_{l A}<A D^{c r}{ }_{A}$ \\
$\mathrm{~B}$ & $A D_{l B} \geq A D^{c r}{ }_{B}$ & $A D_{I B} \geq A D^{c r}{ }_{B}$ & $A D_{I B}<A D^{c r}{ }_{B}$ \\
$\mathrm{C}$ & $A D_{I C}<A D^{c r}{ }_{C}$ & $A D_{I C} \geq A D^{c r}{ }_{C}$ & $A D_{I C}<A D^{c r}{ }_{C}$ \\
\hline
\end{tabular}

The path generated with the expected time (e-path) is different from the path generated with the weighted time (w-path). Considering the expected travel time, it is lower in the e-path than the w-path (about 3\%); considering the weighted travel time, it is higher in the e-path than the w-path. The use of w-path guarantees a bit higher expected travel time but it is more reliable in relation to the flow instability. Paths generated confirm it. Table 3 presents an analysis that correlate clients, zones and time slices. 
Table 2: Optimal routes (sequence of clients and costs).

\begin{tabular}{cclrr}
\hline $\begin{array}{c}\text { Optimization } \\
\text { with }\end{array}$ & Route & \multicolumn{1}{c}{ Client sequence } & $\begin{array}{r}\text { Travel } \\
\text { time }(s)\end{array}$ & $\begin{array}{r}\text { Travel and } \\
\text { delivery }(s)\end{array}$ \\
\hline \multirow{2}{*}{$\begin{array}{c}\text { Expected } \\
\text { costs }(s)\end{array}$} & 1 & $\begin{array}{l}27-26-23-24-3-13-12-2-11-9-4-10-29-28- \\
25\end{array}$ & 804.51 & 6078.51 \\
Total (s) & 2 & $\begin{array}{l}15-18-17-21-19-16-20-22-14-5-6-7-8 \\
\end{array}$ & 1030.09 & 5428.09 \\
\hline \multirow{2}{*}{$\begin{array}{l}\text { Weighted } \\
\text { costs }(s)\end{array}$} & 1 & $\begin{array}{l}28-25-29-24-26-27-23-3-13-12-2-11-9-8- \\
7-6\end{array}$ & 1015.65 & 6655.65 \\
Total $(\mathrm{s})$ & 2 & $22-14-17-20-19-21-16-15-18-5-4-10$ & 1256.34 & 5288.34 \\
\hline
\end{tabular}

Focusing on zone A, there is no change in relation to the number of nodes visited in each time slice. Focusing on zone B, the e-path connects eight nodes (313-12-2-11-9-4-10) in time slice 3 with stable flow conditions and four nodes (56-7-8) in time slice 1 with instable flow conditions. In the same zone, the w-path connects all nodes in time slice 3 with stable flow conditions ( 9 nodes with route 1 and 3 nodes with route 2 ).

Focusing on zone C, the e-path connects three nodes (17-21-19) in time slice 2 with instable flow conditions and six nodes (15-18-16-20-22-14) in time slices 1 and 3 with stable flow conditions. In the same zones, the w-path connects only two nodes in the time slice 2 with instable flow conditions.

Table 3: Scenario 2: visited clients per zone and time slice.

\begin{tabular}{|c|c|c|c|c|c|c|c|c|c|c|}
\hline \multirow{4}{*}{$\begin{array}{l}\text { Expected } \\
\text { costs }\end{array}$} & \multirow{2}{*}{$\begin{array}{l}\text { Zone } \\
\text { Time } \\
\text { slice }\end{array}$} & \multicolumn{3}{|c|}{ A } & \multicolumn{3}{|c|}{ B } & \multicolumn{3}{|c|}{$\mathrm{C}$} \\
\hline & & 1 & 2 & 3 & 1 & 2 & 3 & 1 & 2 & 3 \\
\hline & $\begin{array}{l}\text { Clients of } \\
\text { route } 1\end{array}$ & $\begin{array}{l}27- \\
26\end{array}$ & $\begin{array}{l}23- \\
24\end{array}$ & $\begin{array}{l}29- \\
28- \\
25\end{array}$ & & & $\begin{array}{l}3- \\
13- \\
12- \\
2- \\
11- \\
9-4- \\
10\end{array}$ & & & \\
\hline & $\begin{array}{l}\text { Clients of } \\
\text { route } 2\end{array}$ & & & & $\begin{array}{l}5-6- \\
7-8\end{array}$ & & & $\begin{array}{l}15- \\
18\end{array}$ & $\begin{array}{l}17- \\
21- \\
19\end{array}$ & $\begin{array}{l}16- \\
20- \\
22- \\
14\end{array}$ \\
\hline \multirow[t]{2}{*}{$\begin{array}{l}\text { Weighted } \\
\text { costs }\end{array}$} & $\begin{array}{l}\text { Clients of } \\
\text { route } 1\end{array}$ & $\begin{array}{l}28- \\
25\end{array}$ & $\begin{array}{l}29- \\
24\end{array}$ & $\begin{array}{l}26- \\
27- \\
23\end{array}$ & & & $\begin{array}{l}3- \\
13- \\
12- \\
2- \\
11- \\
9-8- \\
7-6 \\
\end{array}$ & & & \\
\hline & $\begin{array}{l}\text { Clients of } \\
\text { route } 2\end{array}$ & & & & & & $\begin{array}{l}5-4- \\
10\end{array}$ & $\begin{array}{l}22- \\
14\end{array}$ & $\begin{array}{l}17- \\
20\end{array}$ & $\begin{array}{l}19- \\
21- \\
16- \\
15- \\
18\end{array}$ \\
\hline
\end{tabular}




\section{Conclusions}

This paper proposes a formulation of the vehicle routing problem based on the network fundamental diagram. A set of indicators describes the average traffic conditions on portions of the network according to a NFD. The link cost is function of an expected cost (provided by a micro-simulation model) and a weighted cost (related with the cost variance). A cost function to evaluate the link cost is specified, considering in the formulation the density indicators related to the links and the zones. The aim of this formulation is to take into account the reliability of the link cost in terms of average density of link and zone.

Finally, an application in a real dimension network allowed to evaluate the effectiveness of the proposed method. A NFD for each of the three zones is estimated in order to determine the traffic regime (stable or unstable) of the zone in each time slice. A set of clients to be served is considered and a vehicle routing problem is solved by means of a genetic algorithm.

For each time slice, it is evaluated if a zone is in the stable or unstable regime comparing the density of the zone with the critical density according to the estimated NFD. Moreover, for each scenario, the solution obtained by considering the expected link costs is compared with the one obtained by considering the weighted link costs. The preliminary results demonstrate that the optimized routes, in general, tend to avoid heavily congested links and portions of the network.

\section{References}

[1] Laporte, G., Fifty years of vehicle routing. Transportation Science, 43(4) pp. 408-416, 2009.

[2] Dantzig, G. B. \& Ramser, J. H., The truck dispatching problem. Management Science, 6 (1), pp. 80-91, 1959.

[3] Vidal, T., Crainic, T.G., Gendreau, M. \& Prins C.A., Hybrid genetic algorithm with adaptive diversity management for a large class of vehicle routing problems with time-windows, Computers \& Operations Research, 40, pp. 475-489, 2013.

[4] Lei, H., Laporte, G. \& Guo, B., The capacitated vehicle routing problem with stochastic demands and time windows. Computers \& Operations Research, 38, pp. 1775-1783, 2011.

[5] Gauvin, C., Desaulniers, G. \& Gendreau, M., A branch-cut-and-price algorithm for the vehicle routing problem with stochastic demands, Computers \& Operations Research, online 12 April 2014, ISSN 0305-0548.

[6] Marinakis, Y., Iordanidou, G. \& Marinaki, M., Particle swarm optimization for the vehicle routing problem with stochastic demands, Applied Soft Computing, 13, pp. 1693-1704, 2013.

[7] Tasan, A. S. \& Gen, M., A genetic algorithm based approach to vehicle routing problem with simultaneous pick-up and deliveries, Computers \& Industrial Engineering, 62, pp. 755-761, 2012.

[8] Zachariadis, E. E., Tarantilis, C. D. \& Kiranoudis, C. T., An adaptive memory methodology for the vehicle routing problem with simultaneous 
pick-ups and deliveries, European Journal of Operational Research, 202, pp. 401-411, 2010.

[9] Ando, N. \& Taniguchi, E., Travel time reliability in vehicle routing and scheduling with time windows. Networks and Spatial Economics, 6, 293$311,2006$.

[10] Polimeni, A., Russo, F. \& Vitetta, A., Demand and routing models for urban goods movement simulation. European Transport - Trasporti Europei, 46, pp. 3-23, 2010.

[11] Polimeni, A. \& Vitetta, A., Vehicle routing in urban areas: an optimal approach with cost function calibration. Transportmetrica B: Transport Dynamics, 2(1), pp. 1-19, 2014.

[12] Polimeni, A. \& Vitetta, A., Optimising waiting at nodes in time-dependent networks: cost functions and applications. Journal of Optimization Theory and Applications, 156(3), pp. 805-818, 2013.

[13] Barceló, J., Orozco, J. A. \& Grzybowska, H., Making real-time fleet management decisions under time-dependent conditions in urban freight distribution. In Freight Transport Modelling, Ben-Akiva M., Meersman H. \& van de Voorde E. (Eds.), pp. 453-484. Emerald, 2013.

[14] Polimeni, A. \& Vitetta, A., A comparison of vehicle routing approaches with link costs variability: An application for a city logistic plan. WIT Transactions on the Built Environment, 130, pp. 823-834, 2013.

[15] Geroliminis, N. \& Daganzo, C. F., Existence of urban-scale macroscopic fundamental diagrams: Some experimental findings. Transportation Research Part B, 2008.

[16] Geroliminis, N. \& Sun, J., Properties of a well-defined macroscopic fundamental diagram for urban traffic. Transportation Research Part B, 45, pp. 605-617, 2011.

[17] Di Gangi, M., Musolino, G., Russo, F., Velonà, P. \& Vitetta, A., Analysis and comparison of several urban road transportation assignment models in emergency conditions. Sustainable World, 8, pp. 247-257, 2003.

[18] Marcianò, F.A., Musolino, G. \& Vitetta, A., Within-day traffic assignment and signal setting in road evacuation: a procedure with explicit path enumeration. International Journal of Safety and Security Engineering, 3(3), pp. 196-205, 2013.

[19] Praticò, F., Saride, S. \& Puppala, A., Comprehensive life-cycle cost analysis for selection of stabilization alternatives for better performance of lowvolume roads. Transportation Research Record, 2204, 1, pp. 120-129, 2011.

[20] Polimeni, A. \& Vitetta, A., Dynamic vehicle routing in road evacuation: a model for route design. WIT Transactions on the Built Environment, 116, pp. 627-638, 2011.

[21] Briganti, A., Musolino, G. \& Vitetta, A., Simulation on a partitioned urban network: an approach based on network fundamental diagram. WIT Transactions on Ecology and the Environment, 179, (ISSN: 1746-448X, Digital ISSN: 1743-3541), 2014. 
[22] Delfino, G., Iannò, D., Rindone, C. \& Vitetta, A., Stretto di Messina: uno studio della mobilità intermodale per i passeggeri. Alfagi, Reggio Calabria, Italy, 2011.

[23] Dreyfus, S.E., An appraisal of some shortest-path algorithms. Operations Research, 17(3), pp. 395-412, 1969. 